\title{
Domestic Polarization and U.S. Foreign Policy: Ideas, institutions, and policy implications. Ein Bericht zur virtuellen Konferenz am 12. \& 13. November 2020 am Heidelberg Center for American Studies, Universität Heidelberg
}

\section{Gordon M. Friedrichs}

Angenommen: 25. Februar 2021 / Online publiziert: 17. März 2021

(C) Der/die Autor(en) 2021

\section{Einleitung}

Polarisierung ist ein prävalentes Phänomen in der US-amerikanischen Politik und Gesellschaft geworden. Parteiideologische Spaltung, sowohl unter den Wähler*innen als auch zwischen den politischen Eliten, konsumiert die amerikanische Demokratie vollends und hat damit ein politisches System, das auf Kompromissschließung zwischen politischen Rivalen angewiesen ist, in ein System transformiert, welches geprägt ist von politischem Antagonismus, Stillstand und Dysfunktionalität. Diese Entwicklung hat in den vergangenen Jahren expliziten Ausdruck in dem Aufstieg des rechtspopulistischen Präsidenten Donald Trump gefunden sowie in der politischen Gewalt als Reaktion auf die demokratisch-legitime Präsidentschaftswahl 2020, wie beim Sturm auf das Kapitol sichtbar wurde.

Forscher*innen der US-amerikanischen Regierungslehre und Institutionen haben schon seit langem die Ursachen und Implikationen von Polarisierung für den politischen Entscheidungsfindungsprozess untersucht. In den letzten Jahren hat das Thema zudem die Aufmerksamkeit der Internationalen Beziehungen und vergleichenden Außen- und Sicherheitspolitikforschung erhalten. Glaubt man ersten Studien, dann stellt die innenpolitische Polarisierung eine der größten Bedrohungen der US-amerikanischen nationalen Sicherheit dar, unter anderem weil Polarisierung den überparteilichen Konsens für eine Grand Strategy des liberalen Internationalismus untergräbt, die seit dem Ende des Zweiten Weltkriegs als Leitlinie US-amerikanischer Außenpolitik und Stabilitätsanker internationaler Ordnung fungierte.

In einem durch Corona bedingt virtuellen Workshop im November 2020, organisiert von Gordon Friedrichs (Universität Heidelberg) und Jordan Tama (American

Dr. G. M. Friedrichs $(\bowtie)$

Heidelberg Center for American Studies, Ruprecht-Karls Universität Heidelberg,

Hauptstraße 120, 69117 Heidelberg, Deutschland

E-Mail: gfriedrichs@hca.uni-heidelberg.de 
University, Washington, D.C.), trafen mehrere Forscher*innen aus der US-amerikanischen Regierungslehre sowie Außen- und Sicherheitspolitik zusammen, um systematisch die Trendlinien und spezifischen Auswirkungen der innenpolitischen Polarisierung für die US-Außenpolitik und globale Vormachtstellung zu untersuchen. Trotz bestehender außen- und sicherheitspolitischer Herausforderungen, wie dem Aufstieg Chinas, dem assertiven Russland, internationalem Terrorismus, Klimawandel oder Pandemien, eint die an dem Workshop beteiligten Forscher*innen die Annahme, dass Polarisierung die größte derzeitige Bedrohung für den Fortbestand einer internationalen US-amerikanischen Führungsrolle ist. Dies ist, so die Annahme, nicht auf Sicherheitspolitik beschränkt, sondern wirkt sich auch auf andere Politikbereiche aus, wie etwa der internationalen Finanz- und Handelspolitik, der Diplomatie oder auch der amerikanischen Soft Power. Wenngleich wir starke Indizien für den Zusammenhang aus Polarisierung und außenpolitischem Wandel unter der aktuellen Trump-Präsidentschaft erkennen, ist eine zentrale These des Workshops, dass Polarisierung tiefgreifende und nachhaltige Effekte für die an der Gestaltung und Aufrechterhaltung der US-Führungsrolle beteiligten politischen Institutionen hat.

Neben der Tatsache, dass es bislang keine vergleichbare umfassende Studie zu den Effekten von Polarisierung auf die US-amerikanische Außen- und Sicherheitspolitik gibt, brachte der Workshop den Mehrwert, unterschiedliche Perspektiven aus der US-amerikanischen Regierungs- und Institutionenlehre, der Außenpolitikanalyse sowie den Internationalen Beziehungen zusammenzubringen. Es ist beabsichtigt, die Papiere in Form einer Sonderausgabe bei einer englischsprachigen Fachzeitschrift zu veröffentlichen. Insgesamt wurden Papiere zu drei Forschungsfeldern gesammelt und diskutiert.

\section{Sektion I: Polarisierung außenpolitischer Ideen und Präferenzen}

Die Arbeitspapiere in dieser Sektion untersuchten vorrangig den Zusammenhang aus dem Zusammenbruch der ideologischen Mitte in der US-amerikanischen Politik (Kongress) und Gesellschaft (öffentliche Meinung), und der Verteilung (sowie politischen Repräsentation) außenpolitischer Ideen und Präferenzen. Die einzelnen Papiere waren aufgerufen, einen genaueren Blick auf das Ausmaß ideologischer Polarisierung in den USA zu werfen und der Frage nachzugehen, inwiefern sich ideologische Polarisierung in der öffentlichen Meinung und der legislativen Einstellung gegenüber US-Außenpolitik manifestiert hat. Die Ergebnisse dieser Papiere zielen darauf ab, unser Verständnis über außenpolitische Präferenzen, der Rolle von affektiver Polarisierung, dem Wandel der politischen Parteien als Träger außenpolitischer Ideen sowie das Verhältnis von parteipolitischer und ideologischer Polarisierung hinsichtlich internationalen Themenfelder zu erweitern.

Das Papier von Gyung-Ho Jeong (University of British Columbia) und William Bendix (Keene State College) griff letzteren Forschungsstrang in der Polarisierungsforschung auf, der davon ausgeht, dass Kongressabstimmungen zur US-Außenpolitik primär von parteipolitischen Abwägungen und weniger von ideologischen Präferenzen einzelner Abgeordneter motiviert ist, bspw. wenn Kongressabgeordnete Militäreinsätze eines Präsidenten ihrer Partei unterstützen, aber die eines Präsiden- 
ten der Oppositionspartei ablehnen. Jeong und Bendix gingen der Frage nach, ob solche parteipolitischen Entscheidungen sich nur auf wenige Abstimmungen im Bereich Sicherheit reduzieren lassen oder ob dies ein größeres Muster an legislativer Außenpolitikgestaltung darstellt. Um die relative Gewichtung aus parteipolitischer und ideologischer Wahlmotivation zu messen, untersuchten die Autoren Sponsorship und Abstimmungsdaten für Amendments, die es Kongressmitglieder erlauben, Haushaltsmittel für Verteidigung und Entwicklungshilfe zu limitieren (sogenannte Limitation Riders). Für ihren Untersuchungszeitraum 1917-2016 konnten die Autoren nachweisen, dass Ideologie (liberal/konservativ) der ausschlaggebende Faktor bei der Frage ist, ob Kongressabgeordnete den finanziellen Handlungsspielraum des Präsidenten einschränken oder nicht. Demnach nutzen liberale Kongressmitglieder Limitation Riders mehrheitlich bei militärischen Ressourcen, wohingegen konservative Kongressmitglieder vornehmlich humanitäre Ressourcen der Exekutive einschränken.

Jeffrey Lantis (College of Wooster) und Patrick Homan (Dominican University) argumentierten in ihrem Papier, dass Faktionalismus, als Begleiterscheinung parteipolitischer Polarisierung, Innovation im legislativen Außenpolitikgestaltungsprozess fördert. Demnach sind es besonders die sogenannten Free Agents an den ideologisch extremen Rändern ihrer Parteien, wie etwa die Progressive Democrats oder Tea Party-Republikaner, die zunehmend gleiche Außenpolitikpräferenzen teilen und sich gegen das Parteiestablishment auflehnen. Mithilfe einer Plausibilitätsprobe in zwei Fällen der War Powers Resolution unter Barack Obama und Donald Trump untersuchten die Autoren Faktoren, die solche Free Agents zu einem überparteilichen Konsens bewegen.

Sarah Maxey (Loyola University Chicago) ging in ihrem Papier der Frage nach, ob parteipolitische Identität die Bevölkerung davon abhält, dem Präsidenten Unterstützung für den Einsatz des Militärs im Angesicht einer internationalen Krise zuzusichern. Sie argumentierte, dass affektive Polarisierung, also die Ablehnung des politischen Gegners als legitimes Mitglied der Gesellschaft, unterschiedlich über verschiedene Interventionskontexte hinweg wirkt. Mithilfe von Umfrageexperimenten im Zeitraum der Präsidentschaftswahl 2016 zeigte Maxey, dass der Effekt von affektiver Polarisierung bei humanitären Interventionsentscheidungen weniger stark auf die Unterstützung der Öffentlichkeit wirkt als bei anderen Militärinterventionen, und somit der Präsident, trotz Polarisierung der Gesellschaft, durchaus in der Lage ist, überparteiliche Unterstützung in Reaktion auf internationale Krisen zu generieren.

Schließlich untersuchte Dina Smeltz (Chicago Council on Global Affairs) anhand neuster Umfragewerte der amerikanischen Bevölkerung zu außenpolitischen Themen, welche Rolle die Polarisierung der politischen Eliten für die Präferenzbildung der breiten Öffentlichkeit spielt. Damit liefert das Papier einen wertvollen Beitrag hinsichtlich der Frage, ob die Bevölkerung, unabhängig von den politischen Anreizen, die von den politischen Eliten ausgehen, entlang ideologischer und/oder parteipolitischer Dispositionen in Fragen der US-Außenpolitik polarisiert ist - ein anhaltender Streit in der Polarisierungsforschung. 


\section{Sektion II: Innenpolitische Institutionen und Wandel des außenpolitischen Prozesses}

Die zweite Gruppe an Autor*innen nahm die an der US-amerikanischen Außenpolitik beteiligten Institutionen (speziell den Kongress und die Exekutive) und deren potenziellen Wandel im Lichte der Polarisierung genauer in den Blick. Gemeinhin wird davon ausgegangen, dass Polarisierung negative Auswirkungen auf die demokratische Rechenschaftspflicht des politischen Systems und ihren Agenten hat, u. a. weil gewählte Eliten ihre Rolle und politische Verantwortung weniger an ein Gemeingut binden, sondern an ihren ideologisch-homogenen Wahlkreis. Gleichzeitig nutzen Präsidenten zunehmend ihre Exekutivprivilegien, um den politisch blockierten Kongress zu umgehen und ihre Agenda umzusetzen. Die Arbeitspapiere in dieser zweiten Sektion untersuchten vor diesem Hintergrund, inwiefern und in welchem Ausmaß wir institutionelle Polarisierung in der US-Außenpolitik erkennen können, etwa entlang von legislativem Abstimmungsverhalten und Prozessen, der Kollegialität unter Kongressmitgliedern sowie dem Verhältnis der politischen Gewalten.

Das erste Papier von James Bryan und Jordan Tama (beide American University, Washington D.C.) untersuchte die Häufigkeit und den Charakter überparteilichen Konsens im Kongress zu außenpolitischen Abstimmungen im Verhältnis zu innenpolitischen Abstimmungen. Anhand eines Datensets von knapp 3000 Kongressabstimmungen seit dem Ende des Kalten Krieges zeigten die Autoren, dass extreme Polarisierung eher die Ausnahme in der US-Außenpolitik ist und dass der Kongress in außenpolitischen Abstimmungen weniger polarisiert ist als bei innenpolitischen Fragen. Zudem unterschieden die Autoren zwischen verschiedenen Formen überparteilichen Konsens, bspw. gegenüber dem Präsidenten oder überparteilichen Koalitionen.

Shannon Carcelli (University of Maryland) analysierte die veränderte Rolle des Kongresses bei der Vergabe von Entwicklungshilfe. Ausgehend von der Beobachtung, dass aufgrund von legislativer Blockadehaltung der Kongress heutzutage Entwicklungshilfe fast ausschließlich in Form von jährlichen Bewilligungsvorlagen ( $A p$ propriations Bills) vergibt, untersuchte Carcelli den Zusammenhang von Polarisierung und der Zunahme von Limitation Riders, die darauf abzielen, Entwicklungshilfe gesetzgeberisch zu gestalten und zu politisieren. Da Appropriations Bills über den jährlichen Haushalt entscheiden, ist der Rückgriff auf Limitation Riders zwecks Vergabe von Entwicklungshilfe besonders kritisch zu sehen, weil dadurch erkennbar wird, inwiefern Polarisierung die legislativen Prozesse der US-Außenpolitik nachhaltig verändert.

Die veränderte Rolle des Kongresses beschäftigte auch das Papier von Patrick Haney und Bryan Marshall (beide Miami University). Die Autoren attestierten, dass trotz einer breiten gesellschaftlichen Unterstützung für eine globale US-Führungsrolle, der Kongress seine Rolle als Check-and-Balancer der Exekutive in der Außenpolitik weitestgehend aufgegeben hat. Ihre Analyse von Abstimmungsverhalten, Gesetzgebung und Oversight-Funktionen des Kongresses konnte zeigen, in welchem Ausmaß die Legislative politische Autorität im Verhältnis zur Exekutive eingebüßt hat - mit weitreichenden Folgen für die amerikanische Demokratie und verfassungsgemäße Rollenverteilung in der US-Außenpolitik. 
Abschließend präsentierten Sean Theriault und Zachary McGee (beide University of Texas) ein neues Datenset zu Kongressdelegationen ins Ausland. Die Studie ist anschlussfähig an bestehende Forschung zur Kollegialität unter Kongressmitgliedern, die ein substantielles Merkmal des sogenannten Textbook Congress war, in dem überparteilicher Konsens unter anderem durch das freundschaftliche Verhältnis der Abgeordneten hergestellt wurde und sich entlang einer Sachgrundlage für politische Reformen orientierte. Die Autoren zeigten entlang einer Längsschnittanalyse, dass Kongressdelegationen seit 1977 und der Zunahme an Polarisierung immer weniger aus Mitgliedern beider Parteien bestehen, was wiederum Folgen für die Wahrnehmung der beiden Parteien von US-Außenpolitik hat als auch für deren Verhalten im außenpolitischen Gesetzgebungsprozess.

\section{Sektion III: Polarisierung und die Implementierung von US- Außenpolitik}

Die dritte Gruppe an Arbeitspapieren konzentrierte sich auf die Auswirkungen innenpolitischer Polarisierung auf die Form und Qualität US-amerikanischer Außenpolitik. Die Ergebnisse der Papiere trugen damit zu der generellen Frage bei, ob und inwiefern Polarisierung grundsätzlich die US-amerikanische internationale Vormachtstellung unterwandert. Dabei nahmen die Autor*innen eine ganze Spannbreite an Politikfeldern in den Blick, wie etwa Rüstungskontrollpolitik, Handelspolitik, Militärinterventionen sowie Allianzpolitik.

Zunächst untersuchte Florian Böller (Technische Universität Kaiserslautern) den Einfluss von Polarisierung auf die Ratifikationsfähigkeit internationaler Abrüstungsverträge. Mithilfe einer Qualitative Comparative Analysis (QCA) untersuchte er 23 Rüstungskontrollverträge mit US-Beteiligung seit 1963. Die Analyse konnte zeigen, dass die relative Stärke einer konservativen Gruppe an Senatoren, die internationalen Verträgen skeptisch gegenübersteht, maßgeblichen Anteil an der Abnahme ratifizierter Rüstungskontrollverträge der USA hat. Zudem geht das Papier in einer detaillierten Einzelfallstudie zur US-Rüstungskontrollpolitik unter Trump weiteren Erklärungsfaktoren nach.

Das Papier von Gordon Friedrichs (Universität Heidelberg) untersuchte, inwiefern sich innerparteiliche Polarisierung als Produkt zwischenparteilicher Polarisierung auf die US-Handelspolitik auswirkt. Anhand eines neuen Datensatzes bestehend aus Co-Sponsorship-Allianzen bei eingebrachten Gesetzesentwürfen und Briefen an die Exekutive operationalisierte Friedrichs die Außenhandelspräferenzen von Kongressmitgliedern in zwei Fallstudien: dem Transpazifischen Partnerschaftsabkommen und dem US-Mexico-Canada-Agreement. Die Studie konnte zeigen, dass der Grad an innerparteilicher Polarisierung - zwischen Freihandelsbefürworter*innen und Protektionist*innen sowie Establishment- und Anti-Establishment-Ideologien entscheidend Einfluss auf die Ausgestaltung und Ratifikation von Handelsabkommen mit anderen Staaten hat.

Carrie Lee (United States Air War College) ging der Frage nach, inwiefern und vor allem wann sich politische Polarisierung auf das Verhalten der USA während eines Militäreinsatzes auswirkt. Das Papier von Lee untersuchte speziell, wie sich 
unterschiedliche Opfer-Sensibilitätsniveaus entlang der Parteilinie auf die Anreizstrukturen von Präsidenten auswirken, Kriege zu führen. Auf Grundlage einer repräsentativen Umfrage unter 2500 Amerikaner*innen schlussfolgerte sie, dass konservative Wähler*innen weitaus weniger opfersensibel sind, speziell bei konventioneller Kriegsführung, was auf eine größere Bereitschaft von republikanischen Präsidenten schließen lässt, offensive Kriegsführung zu betreiben, die darauf abzielt, Erfolge auf dem Schlachtfeld unter Beweis zu stellen. Im Gegenzug fand Lee unter demokratischen Wähler*innen eine höhere Opferaversität, sowohl bei asymmetrischer als auch konventioneller Kriegsführung. Demzufolge kann zunehmende bzw. anhaltende Polarisierung die Kriegsführung der USA nachhaltig beeinflussen.

Schließlich untersuchte Rachel Myrick (Duke University), inwiefern parteipolitische Polarisierung die britische Wahrnehmung der US-Sicherheitsgarantien und internationale Führungsrolle beeinflusst. Anhand eines Umfrageexperiments unter 2000 Brit*innen demonstrierte sie, dass Polarisierung in den USA Alliierte zwar weniger die derzeitigen unmittelbaren US-Sicherheitsgarantien anzweifeln lässt, jedoch reduziert es die Bereitschaft von Verbündeten, auch zukünftig eine enge Partnerschaft mit den USA einzugehen. Somit produziert innenpolitische Polarisierung langfristig internationale Unsicherheit über die US-Außenpolitik.

\section{Allgemeiner Forschungsbeitrag}

Insgesamt leisten die Beiträge wichtige, umfangreiche Erkenntnisse über das Ausmaß und die Wirkkraft innenpolitischer Polarisierung auf die US-amerikanische Außenpolitik. Dabei greifen sie auf ein breites Spektrum an Methoden der Außenpolitikanalyse und vergleichenden Regierungslehre zurück. Drei generelle Erkenntnisse sind abschließend festzuhalten:

Erstens, ideologische Polarisierung beweist sich als weitaus wirkmächtiger als parteipolitische Polarisierung hinsichtlich der außenpolitischen Präferenzen politischer Eliten und der Öffentlichkeit. Dadurch ergeben sich in einigen Fällen neue Koalitionen für Außenpolitiken, die zu Teilen von ideologisch extremen Faktionen innerhalb der Parteien propagiert werden und eine Pro-/Anti-Establishment-Konfliktlinie abbilden.

Zweitens zeigt das Verhalten von Kongressmitgliedern sowie der Exekutive, dass mit Zunahme ideologischer Polarisierung eine Reinterpretation der bestehenden Normen des außenpolitischen Gestaltungs- und Entscheidungsprozesses verbunden ist. Das drückt sich unmittelbar in einer Schwächung der Kontrollfunktion des Kongresses aus, hat aber darüber hinaus in den empirischen Fallstudien aufgezeigt, welche qualitativen Veränderungen (Richtung, Intensität, Umfang) damit für die US-amerikanische Außenpolitik verbunden sind. Folglich ist zu erwarten, dass die USAußenpolitik in Zukunft deutlich stärker von den Mechanismen der Politisierung im Inneren, zwecks Marginalisierung des politischen Rivalen und der disproportionalen Repräsentation marginaler Wählergruppen, bestimmt werden wird, als weniger von einem Leitmotiv, dass sich am internationalen Staatensystem orientiert.

Drittens kann festgehalten werden, dass Polarisierung zwar nicht zwingend die Kapazitäten der USA vermindert, jedoch die Effektivität US-amerikanischer Au- 
Benpolitik nachhaltig einschränkt. Das zeigt sich nicht nur bei der internationalen Vertragspolitik, die sowohl unzuverlässiger wird als auch seltener Ergebnisse produziert, sondern auch bei der Interventions- und Entwicklungspolitik, die größere politische und materielle Opportunitätskosten mit sich bringen.

Aufgrund dieser Entwicklungen läuft die US-Außenpolitik Gefahr, internationale Unterstützung im Sinne der Lastenteilung und institutionalisierter Kooperation zur Maximierung von geteilten Gewinnen zu verlieren. Das lässt darauf schließen, dass die nationalen Strukturbedingungen der US-Demokratie internationale Ordnungspolitiken langfristig verschieben könnten - weg von einer kooperationsorientierten Ordnung hin zu einer transaktionalistischen, in der Staaten nach kurzfristigen Gewinnen streben, weil Wahlen kein Vertrauen mehr in eine regelbasierte US-amerikanische Außenpolitik herstellen können. Die gesammelten Beiträge für diesen Workshop haben eine wichtige Grundlage für eine weitere wissenschaftliche Auseinandersetzung mit diesen Phänomenen der US-Außenpolitik gelegt.

Funding Open Access funding enabled and organized by Projekt DEAL.

Open Access Dieser Artikel wird unter der Creative Commons Namensnennung 4.0 International Lizenz veröffentlicht, welche die Nutzung, Vervielfältigung, Bearbeitung, Verbreitung und Wiedergabe in jeglichem Medium und Format erlaubt, sofern Sie den/die ursprünglichen Autor(en) und die Quelle ordnungsgemäß nennen, einen Link zur Creative Commons Lizenz beifügen und angeben, ob Änderungen vorgenommen wurden.

Die in diesem Artikel enthaltenen Bilder und sonstiges Drittmaterial unterliegen ebenfalls der genannten Creative Commons Lizenz, sofern sich aus der Abbildungslegende nichts anderes ergibt. Sofern das betreffende Material nicht unter der genannten Creative Commons Lizenz steht und die betreffende Handlung nicht nach gesetzlichen Vorschriften erlaubt ist, ist für die oben aufgeführten Weiterverwendungen des Materials die Einwilligung des jeweiligen Rechteinhabers einzuholen.

Weitere Details zur Lizenz entnehmen Sie bitte der Lizenzinformation auf http://creativecommons.org/ licenses/by/4.0/deed.de. 